\title{
Event Related Potentials and its role as Endophenotypes in Psychiatry
}

\author{
Dr. Roshan Khanande ${ }^{1}$, Mrs. Bhoomika Sachacher ${ }^{2}$, Dr. B. Das ${ }^{3}$, Dr. S. H. Nizamie ${ }^{4}$
}

${ }^{1}$ MD Psychiatry, Senior Resident, Central Institute of Psychiatry, Kanke, Ranchi, Jharkhand, ${ }^{2}$ PHD Scholar, Central Institute of Psychiatry, Kanke, Ranchi, Jharkhand, ${ }^{3}$ MD Psychiatry, Associate Professor, Central Institute of Psychiatry, Kanke, Ranchi, Jharkhand, ${ }^{4} \mathrm{MD}$, DPM, Professor of Excellence, Central Institute of Psychiatry, Kanke, Ranchi, Jharkhand

\section{ABSTRACT :}

Familial segregation is a norm rather than an exception in psychiatry. Genetic research has failed to provide much anticipated unanimous, if not awaited answers to the complex predispositions to psychiatric disorders. Hence, the concept of endophenotype took birth. Endophenotype is an attribute which is hidden phenotype, which is described as internal phenotypes discoverable by biochemical test or microscopic examination. This concept was immediately picked up by psychiatry, giving a totally new direction to the search for root cause of various psychiatric disorders.Cognitive deterioration is inseparable from psychiatric disorders, known since dawn of psychiatry. However, cognitive deficits in FDRs (First Degree Relatives) of psychiatric patients were one of the first endophenotypes noted. Event Related Potential (ERP), a science known to be electrophysiological counterpart of various cognitive processes, was soon incorporated in search of endophenotypes in psychiatry. In schizophrenia reduced amplitude of MMN(Mismatch negativity), impaired suppression of P50 and reduced amplitude of P300; reduced P300 amplitude, impaired P50 suppression in Bipolar Affective Disorders; reduced P300 amplitude in substance dependence disorders have been reported to have endophenotypic values. Various other ERP waves such as $\mathrm{CNV}, \mathrm{N} 400$ have been investigated but no conclusive reports are out yet. The ultimate goal of this science is to search for genes governing the cognitive processes responsible for ERP waves identified as Endophenotypes for specific psychiatric disorders, and the search has just started.

Keywords : Event Related Potential, Endophenotype, Psychiatric Disorder, Mismatch negativity, P 300, P 50, CNV

\section{INTRODUCTION}

Familial segregation is often seen in various psychiatric disorders. There is strong evidence that these disorders have a genetic basis, withhigh levels of monozygotic twin concordance, firstdegree relatives (FDRs) showing increased rates of occurrence of these disorders as compared with the normal population (Gottesman\& Gould, 2003). These evidences motivated researchers to find genes responsible through techniques of molecular biology such as polymerase chain reaction (PCR) and DNA

Address for Correspondence

Dr. Roshan Khanande, Senior Resident, Central Institute of

Psychiatry, Kanke, Ranchi

Email : roshankhanande@gmail.com sequencing, with the hope that the day won't be far when early intervention and permanent cure of these debilitating disorders would be possible. Candidate gene association studies have not yet provided consistent evidence to confirm specific genes involvement(Wang et al, 2010). The quest to find genetic cure for psychiatric disorders or for that matter prediction of occurrence seemingly remain out of human reach (Gottesman\& Gould, 2003).

Gottesman and Shields (1973) gave the term endophenotypes or hidden phenotype, which they described as internal phenotypes discoverable by a "biochemical test or microscopic examination." The concept of endophenotype was readily adapted 
by psychiatry as it seemed to fill the gap between syndromal picture of psychiatric disorders and the elusive disease processes. Search for endophenotype in different psychiatric disorders started with schizophrenia and BPAD (Gottesman\& Gould, 2003). To find out endophenotypic value of the various cognitive deficits,patients and FDRs (First Degree Relatives) of patients with psychiatric illnesses were investigated on tests for different cognitive domains revealing deficits.For example, patients with OCD along with their FDRs showed deficits in areas of response inhibition andset shifting (Chamberlain et al, 2007; Rajender et al, 2011). As it is known that Event Related Potential (ERP) waveforms identify themselves with various cognitive processes such as P50 which is the indicator of attention to sensory inputs and P300 is the marker of processing speed (Patrick et al, 2006), researchers started studying these and various other waveforms in patients with psychiatric disorders and their FDRs (Gottesman\& Gould, 2003). Present article has tried to give an overview of different ERP waveforms and electrophysiological endophenotypes in psychiatry.

\section{WHAT IS EVENT RELATED POTENTIAL (ERP)? AND WHY IT IS TO BE STUDIED?}

Event related potentials (ERP) are very small voltages generated in the brain structures in response to specific events or stimuli (Blackwood \& Muir, 1990). They are EEG changes that are time locked to sensory, motor or cognitive events that provide safe and non invasive approach to study psychophysiological correlates of mental processes.Event-related potentials can be elicited by a wide variety of sensory, cognitive or motor events (Peterson et al, 1995). ERP in humans can be divided into two categories. The early waves or components peaking roughly within first 100 milliseconds after stimulus are termed 'sensory' or 'exogenous' as they depend largely on the physical parameters of the stimulus. In contrast, ERPs generated in later parts reflect the manner in which the subject evaluates the stimulus and are termed 'cognitive' or 'endogenous'
ERPs as they examine information processing (Pritchard, 1986).

ERP waveforms are described in terms of positive and negative peaks. Generally, the labeling refers to the sequence in which the peak occurs and to its polarity. For example, N2 would refer to second negative peak in the waveform. The naming system of ERP can also identify the positive and negative peaks by their latency (usually defined as time from the stimulus onset). N100, for example, denotes the negative peak occurring $100 \mathrm{msec}$ following the stimulus onset (Key, 2005). These positive or negative peaks denote amplitude which is defined as the voltage difference between a pre-stimulus baseline and the largest peak of the ERP waveform within a latency range for the wave (Polich\& Hoffman, 1999).

This science of studying human behavior through electrical activity in the brain has come a long way. The first clinical applications for evoked potentials was performed by Halliday et al ( 1973) who discovered abnormal visual evoked potentials in multiple sclerosis patients. At the same time, Jewett reported recordable early electrical potentials to auditory stimulation (Jewett, 1970). Early somatosensory potentials were demonstrated that originated from subcortical regions of the nervous system (Chiappa, 1990).

These techniques over the past few decades have extensively been used in evaluating psychiatric disorders as they exhibit abnormalities in cognition and behavior. It has been established that various waveformsidentifythemselveswithvariouscognitive processes such as P50 is the indicator of attention to sensory inputs and P300 is the marker of processing speed (Patrick et al, 2006). It is a well known fact that individuals with psychiatric disorders show deficits in various cognitive processes for example attention and information processing deficits in schizophrenia (Rao, 2004; Cadenhead et al, 1997). With the help of these techniques, neuropsychological deficits in a 
psychiatric disorder can be understood in terms of changes in the waveforms identifying them. These techniques not only help in monitoring deficits over time but also can establish improvements by the remediation practiced.

\section{TYPES OF ERP-MODALITIES}

ERP can be recorded in all sensory modalities namely auditory, visual, somatosensory or it can be multimodal, involving more than one modality.

ERP protocol can be active or passive -

Active protocol-Processing of targetstimulus by patient is given some external means of observation eg. Pressing a button.

Passive protocol- In this protocol, no external mechanism to verify patients' target stimulus is given.

Task related ERP- ERP is recorded while patient performs some cognitive task.

\section{DIFFERENT ERP WAVEFORMS AND THEIR COGNITIVE REPRESENTATION}

\section{P 50}

Amount of attenuation in the neural response to the second of two identical stimuli indexes the strength of the inhibitory pathway in the brain (Eccles, 1969). This paradigm has been adapted in psychophysiological research as a test of sensory gating principally through the study of P50 waveform. Sensory gating is crucial to an individual's ability to selectively attend to salient stimuli and ignore redundant, repetitive, or trivial information, protecting the brain from information overflow (Light \&Braff, 2003).

\section{P300 (P3)}

Since its discoveryP3 has been the major component of research in the field of ERP. For auditory stimuli, the latency range is between $250-400 \mathrm{msec}$ for most adult subjects between 20 and 70 years of age
(Polich, 1991). The latency is usually interpreted as the speed of stimulus classification resulting from discrimination of one event from another. Shorter latencies indicate superior mental performance relative to longer latencies. Greater attention produces larger P3 waves (Israel et al, 1980).A wide variety of paradigms have been used to elicit the P3 of which the 'oddball' paradigm is the most utilized. As the P3 wave is large, it is easy to identify in most subjects.

\section{CONTINGENT NEGATIVE VARIATION (CNV)}

Grey Walter (1964) reported slow surface negativity that depends upon the association or contingency of two successful stimuli and named it contingent negative variation. $\mathrm{CNV}$ can be elicited by a standard reaction time paradigm (S1-S2-motor response) or only by paired stimuli without any motor response (S1-S2 paradigm). A first stimulus (S1) serves as a preparatory signal for an imperative stimulus (S2) to which the subject must make a response. Based on presence or absence of S1, S2 and motor response, four sources of negativity can be identified -

1) Early negativity in response to S1 (Orienting response or O wave);

2) Early negativity in expectancy of S2 (expectancy wave or E wave);

3) Late negativity in anticipation of processing S2 (Attention wave or A wave);

4) Late negativity in preparation for motor response to S2 (Readiness Potential or RP wave) (Kornhuber \& Deecke, 1978).

\section{N100 (N1)}

A negative deflection peaking between 90 and $200 \mathrm{msec}$ after the onset of stimulus, is observed when an unexpected stimulus is presented. It is an orienting response or a "matching process," that is, whenever a stimulus is presented, it is matched with previously experienced stimuli and if it does not match produces the wave. It has maximum amplitude over $\mathrm{Cz}$ and is therefore also called "vertex potential" (Sur \& Sinha, 2009). 


\section{N200 (N2)}

N2 is a negative deflection peaking at about 200 msec after presentation of a stimulus. There are 3 components of the N200 waveform-

\section{N2a/MISMATCH NEGATIVITY (MMN)}

MMN is a negative component which is elicited by any discriminable change (Näätänenet al, 1998) in a repetitive background of auditory stimulation (Winkler et al, 1996). Its latency lies between 100msec - 250msec. MMN represents the brain's automatic process involved in encoding of the stimulus difference or change

\section{$\mathbf{N} 2 b$}

It is slightly later in latency than the N2a and appears when changes in physical property of the stimulus are task relevant.

\section{N2C}

It is the classification N2, elicited when classification of disparate stimuli is needed.

\section{N400}

It is a negative wave first described by Kutas et al (1984) in the context of semantic incongruity. The N400 was quantified as the average amplitude difference between the congruent and incongruent ERPs in the region 300-600 msec post-stimulus (Kutas et al, 1984). Topographically N400 is generated by the right hemisphere. N400 is inversely related to the expectancy of a given word to end a sentence.

\section{P600}

In the domain of language processing, a P600 effect occurs to sentences that (a) contain a syntactic violation, (b) have a nonpreferred syntactic structure or (c) have a complex syntactic structure. It is a potential with a positive polarity, starting at about $500 \mathrm{~ms}$ and typically extending up to at least 800 msec (Osterhout\& Holcomb, 1992).

\section{ENDOPHENOTYPE CONCEPT}

The term "genetics" was provided by William Bateson in 1902. In 1911, the present day distinction, which we now take for granted between the concept of "genotype" and the concept of "phenotype" was provided by the Danish botanist Wilhelm Johannsen. His research led to the finding that a genotype can give rise to variety of phenotypes and a phenotype can be an external representation of different genotypes. Genotypes, which can be measured with techniques of molecular biology, are often useful as probabilistic prognosticators of a disease (Gottesman \& Gould, 2003).

As we know it is difficult to pinpoint a single gene in most of the psychiatric disorders, the inheritance of psychiatric disorders remains polygenic mostly. At first, polygenic model of inheritance was ascribed to schizophrenia and while describing genetic studies of schizophrenia Gottesman and Shields (1973) gave the term endophenotypes which they described as internal phenotypes discoverable by a "biochemical test or microscopic examination." The concept of endophenotype was readily adapted by psychiatry as it seemed to fill the gap between syndromal picture of psychiatric disorders and the elusive disease processes. The argument in favor of studying endophenotypes is, the number of genes representing a neurophysiological, biochemical, endocrinological, neuroanatomical, cognitive or neuropsychological attribute, which is associated with illness in the population, which is heritable, non state dependent, found in non affected relatives and occur more often in families of an affected individual than general population, will definitely be less than the number of genes representing complex behavioral syndromes. So to conclude, the endophenotypes are measurable components unseen by the unaided eye along the pathway between disease and distal genotype (Gottesman\& Gould, 2003; Leboyer et al, 1998). 


\section{COGNITIVE PROCESSES, ERP, AND ENDOPHENOTYPE}

Patients with schizophrenia and their FDRs have been shown to have deficits in areas of cognition such asimmediate and delayed memory, and executive function (Hoff et al, 2005; Myles-Worsley et al, 2007). Similarly, BPAD patients and their FDRs have shown deficits on tests for executive functions (Frangou et al, 2005a; Bora et al, 2008). Metaanalytic study by Bora et al (2009) demonstrated that impaired response inhibition might be the most prominent cognitive endophenotype of BPAD. Slowing in cognitive functions has been postulated as a candidate endophenotype for depression (Hasler et al, 2004). Motor impulsivity (the inability to delay or inhibit a proponent motor response) and cognitive impulsivity (impulsive decision making such as the inability to shift sets) is found to be impaired in families of BPD patients (Siddiqui et al, 2011).

Cognitive endophenotypes have been established by various researchers in psychiatric disorders. As depicted above in the present review it is clear that various ERP waveforms are electrophysiological counterparts of underlying sensory and cognitive processes. Now it is logical to see ERP waveforms as endophenotypes for psychiatric disorders. Present review looks at electrophysiological endophenotypes in psychiatry.

Electrophysiological Endophenotype in Different Psychiatric Disorders

\section{SCHIZOPHRENIA}

Electrophysiological studies have also revealed some of the candidate endophenotypes in schizophrenia. A reduction in amplitude of MMN component of event related potential has been proposed as an endophenotype for schizophrenia and was first reported by Shelley et al (1991). Further research in this area gave similar findings. Reduced amplitude of MMN is also present in first-degree relatives (Jessen et al, 2001; Michie et al, 2002) and in at-risk children
(Schreiber et al, 1992) of patients with schizophrenia. Impaired suppression of the $\mathrm{P} 50$ has been proposed as an endophenotype for schizophrenia and was first reported by Adler et al (1982). Similar findings have also been reported by Clementz et al (1998), Waldo et al $(1991,2000)$. Amplitude reduction of the P300 has been proposed as an endophenotype and was first reported by Roth and Cannon (1972). It has also been reported in unaffected first-degree relatives (Frangou et al, 1997; Turetsky et al, 2000; Price et al, 2006), siblings (Karoumi et al, 2000; Saitoh et al, 1984), and in at-risk children (Schreiber et al, 1992) of patients with schizophrenia.

\section{BIPOLAR AFFECTIVE DISORDER (BPAD)}

After schizophrenia most sought after disorder for establishment for endophenotypes is bipolar affective disorder (BPAD). There are two family reports, one demonstrating both reduced P300 amplitude and prolonged latency in relatives of patients with BPAD (Pierson et al, 2000) and the other finding was prolonged latency in relatives (Schulze et al, 2005). Study investigating P50 suppression in relatives of bipolar patients found impairment in both patients and relatives, suggesting that P50 suppression may be associated with genetic liability to psychotic bipolar illness (Schulze et al, 2007). One 2007 study conducted on 10 discordant monozygotic (MZ) bipolar twin pairs, six concordant MZ bipolar twin pairs and 78 control twin pairs by Hall et al found smaller P300 amplitude and impaired P50 suppression in bipolar twin pairs. These findings were similar to previous studies in BPAD.

\section{DEPRESSION}

Thus cognitive function may be a candidate endophenotype for depression (Hasler et al, 2004). There is no clear consensus over electrophysiological endophenotype in depression, but studies have shown prolonged P300 latency and lower P300 amplitude (Singh et al, 2000; Bruder et al, 1991) in patients with depression. P50 suppression deficits have also been reported in patients with depression (Wang et al, 2009). 


\section{OBSESSIVE COMPULSIVE DISORDER (OCD)}

There has been no study pinpointing electrophysiological endophenotype in $\mathrm{OCD}$, but various researchers have found short P300 latency and small P300 amplitude (Kivircik et al, 2003; Kim et al, 2003; Sanz et al, 2001). Impaired P50 suppression (Hashimoto et al, 2008; Nanbu et al, 2010) and higher amplitude of the 'late' component CNV in OCD patients (Sartory\& Master, 1984) have been reported.

\section{OTHER ANXIETY DISORDERS}

Except $O C D$, other anxiety disorders are not yet investigated in detail for cognitive endophenotypes. Autonomic hyperactivity, heart rate variability and startled responses are commonly present in anxiety disorders. In Post Traumatic Stress Disorder (PTSD), psychological variables like memory problems have been reported (Broekmana et al, 2007; Siddiqui et al, 2011). Impaired sensory gating has also been reported in PTSD (Metzger et al, 2002) and Panic disorder (Ghisolfi et al, 2006).

\section{PERSONALITY DISORDERS}

Both twin and family studies, including adoptive studies, strongly suggest a genetic component for personality and personality disorder diagnosis. Twin studies, including monozygotic twins reared together and apart, support a robust genetic influence on personality dimensions (Pedersen et al, 1991; Tellegen et al, 1988). Suppression of the P50 event related potential and prepulse inhibition, both related to inhibitory functions, were found impaired in schizotypal personality disorder (Cadenhead et al, 2002). Impulsivity is one of the characteristic of Borderline Personality Disorder (BPD). Motor impulsivity (the inability to delay or inhibit a proponent motor response) and cognitive impulsivity (impulsive decision making such as the inability to shift sets) is found to be impaired in families of BPD patients, on tests like GO/NO GO task and CPT etc. respectively (Winstanley et al, 2004; Siever, 2005; Siddiqui et al, 2011). Other personalities are not being investigated yet for cognitive or electrophysiological endophenotypes.

\section{SUBSTANCE DEPENDENCE}

Substance dependence is no short of genetic predisposition and dilemma associated with research claiming varied genetic foci (Gottesman\& Gould, 2003). As for other psychiatric disorders, substance dependence disorders have also been reported for cognitive deficits. Researchers have also reported response inhibition deficits in FDRs of alcohol dependence patients (Kamarajan et al, 2005). Cognitive endophenotypes have paved way for search for electrophysiological endophenotypes. Low amplitude P300 waveform has been consistently reported in alcohol dependence syndrome (ADS), high risk individuals, long term abstinent ADS individuals and FDRs of individuals with ADS. N400 component, an electrophysiological correlate of semantic priming, have also been reported to have endophenotypic value as individuals with ADS and high risk individuals showed less attenuation on lexical decision task as compared to normal controls (Roopesh et al, 2010). So, low P300 amplitude and less attenuated N400areprobableendophenotypes for alcohol dependence.

Substances other than alcohol have also been studied. Meta-analysis by Euser et al (2012) has concluded reduced P300 as an endophenotype for substance use disorders as a whole. Other ERP waveforms are being currently investigated for different substance use disorders for their endophenotype status.

\section{FUTURE DIRECTIONS}

This area concerning ERP and its implications has given a whole new direction in the search for endophenotypes for various psychiatric disorders. Still, a lot has to be done as there is dearth of research for disorders like personality disorders on one hand and plenty of research, though at times conflicting for some of the disorders like schizophrenia and depression. This science has long way to go in search 
for specific ERP waveforms concerning specific psychiatric disorders. Stringent inclusion criterion, controlling drug status and exploring different wave forms might pave way for identifying specific electrophysiological endophenotype and would ultimately help in locating particular gene/gene cluster responsible for a psychiatric disorder.

\section{CONCLUSION}

Twin and family studies have shown the importance of biological variation in psychiatric disorders. Heritability estimates vary from $50 \%$ to $80 \%$ for cognitive disorders, such as schizophrenia, attention deficit hyperactivity disorder and autism, and from $40 \%$ to $65 \%$ for affective disorders, such as major depression, anxiety disorders and substance abuse. Pinpointing the actual genetic variants responsible for this heritability has proven difficult, even in the recent wave of genome-wide association studies. Brain endophenotypes derived from electroencephalography (EEG) have been proposed as a way to support gene-finding efforts. A variety of EEG and event-related-potential endophenotypes are linked to psychiatric disorders, and twin studies have shown a striking genetic contribution to these endophenotypes. However, the clear need for very large sample sizes strongly limits the usefulness of EEG endophenotypes in gene-finding studies. EEG endophenotypes are far more promising as tools to make sense of candidate genetic variants that derive from association studies. EEG endophenotypes can help us understand where in the brain, in which stage and during what type of information processing these genetic variants have a role. Such testing can be done in the more modest samples that are feasible for EEG research. With increased understanding of how genes affect the brain, combinations of genetic risk scores and brain endophenotypes may become part of the future classification of psychiatric disorders.

\section{REFERENCES}

Adler, L.E., Pachtman, E., Franks, R.D., et al (1982) Neurophysiological evidence for a defect in neuronal mechanisms involved in sensory gating in schizophrenia. Biological Psychiatry, 17, 639-654.
Andrew, c. \& Fein, G. (2010) Event-related oscillations versus event-related potentials in a P300 task as biomarkers for alcoholism. Alcoholism, Clinical and experimental Research, 4, 669-680.

Bateson, W. (1902) Mendel's principles of heredity : A defence. Cambridge University Press, Cambridge.

Blackwood, D.H.R. \& Muir, W.J. (1990) Cognitive brain potentials and their application. The British Journal of Psychiatry, 157, 96-101.

Bora, E., Yucel, M., Pantelis, C. (2009) Cognitive endophenotypes of bipolar disorder : A meta-analysis of neuropsychological deficits in euthymic patients and their first-degree relatives. Journal of Affective Disorders, 113, 1-20.

Bora, E., Vahip, S., Akdeniz, F., et al (2008) Executive and verbal working memory dysfunction in first- degree relatives of patients with bipolar disorder. Psychiatry Research, 161, 318-324.

Broekmana, B.F.P., Olffb, M., Boer, F. (2007) The genetic background to PTSD. Neuroscience and Biobehavioral Reviews, 31, 348-362.

Bruder, G.E., Towey, J.P., Stewart, J.W., et al (1991) Event-related potentials in depression: influence of task, stimulus hemifield and clinical features on P3 latency. Biological Psychiatry, 30, 233-246.

Cadenhead, K.S., Geyer, M.A., Butler, R.W., et al (1997) Information processing deficits of schizophrenia patients : Relationship to clinical ratings, gender and medication status. Schizophrenia Research, 28, 51-62.

Cadenhead, K.S., Light, G.A., Geyer, M.A., et al (2002 Neurobiological Measures of Schizotypal Personality Disorder: Defining an Inhibitory Endophenotype? American Journal of Psychiatry, 159, 869-871.

Chamberlain, S.R., Fineberg, N.A., Menzies, L., et al (2007) Impaired Cognitive Flexibility and Motor Inhibition in Unaffected First-Degree Relatives of Patients with ObsessiveCompulsive Disorder. American Journal of Psychiatry, 164, 335-338.

Chiappa, K.H. (1990) Evoked Potentials in Clinical medicine, 2nd ed., New York, Raven Press.

Clementz, B.A., Geyer, M.A., Braff, D.L. (1998) Poor P50 suppression among schizophrenia patients and their firstdegree biological relatives. American Journal of Psychiatry, $155,1691-1694$.

Eccles, J. (1969) The Inhibitory Pathways of the Central Nervous System. University Press, Liverpool, UK.

Eusera, A.S., Arends, L.R., Evans B.E., et al (2012) The P300 eventrelated brain potential as a neurobiological endophenotype forsubstance use disorders: A meta-analytic investigation. Neuroscience andBiobehavioral Reviews, 36, 572-603.

Frangou, S., Haldane, M., Roddy, M., et al (2005a) Evidence for deficits in tasks of ventral, but not dorsal, prefrontal executive function as an endophenotypic marker for bipolar disorder. Biological Psychiatry, 58, 838-839.

Frangou, S., Sharma, T., Alarcon, G., et al (1997) The Maudsley Family Study, II : Endogenous event-related potentials in familial schizophrenia. Schizophrenia Research, 23, 45-53. 
Ghisolfi, E.S., Heldt, E., Zanardo, A.P., et al (2006) P50 sensory gating in panic disorder. Journal of Psychiatric Research, 40, 535-540.

Gottesman, I.I. \& Gould, T.D. (2003) The Endophenotype Concept in Psychiatry : Etymology and Strategic Intentions. American Journal of Psychiatry, 160, 636-645.

Gottesman, I.I. \& Shields, J. (1973) Genetic theorizing and schizophrenia. British Journal of Psychiatry, 122, 15-30.

Halliday, A.M., McDonald, W.I., Mushin, J. (1973) Visual evoked responses in the diagnosis of multiple sclerosis. British Medical Journal, 4, 661-664.

Hashimoto,T.,Shimizu,E.,Koike,K.,etal(2008)Deficitsinauditory P50 inhibition in obsessive-compulsive disorder. Progress in Neuro-Psychopharmacology and Biological Psychiatry, $32,288-296$

Hasler, G., Drevets, W.C., Manji, H.K., et al (2004) Discovering Endophenotypes for Major Depression Neuropsychopharmacology, 29, 1765-1781.

Hoff, A.L., Svetina, C., Maruizio, A.M., et al (2005) Familial cognitive deficits in schizophrenia. American Journal of Medical Genetics Part B : Neuropsychiatric Genetics, 133, 43-49.

Israel, J.B., Wickens, C.D., Chesney, G.L., et al (1980) The Event Related Brain Potential as an index of display-monitoring workload. Human Factors, 22, 211-224.

Jessen, F., Fries, T., Kucharski, C., et al (2001) Amplitude reduction of the mismatch negativity in first-degree relatives of patients with schizophrenia. Neuroscience Letters, 309, 185-188.

Jewett, D.L. (1970) Volume-conducted potentials in response to auditory stimuli as detected by averaging in the cat. Electroencephalography and Clinical Neurophysiology, 28, 609-618.

Kamarajan, C., Porjesz, B., Jones, K.A., et al (2005) Spatialanatomical mapping of NoGo-P3 in the offspring of alcoholics: evidence of cognitive and neural disinhibition as a risk for alcoholism. Clinical Neurophysiology, 116, 1049-1061.

Karoumi, B., Laurent, A., Rosenfeld, F., et al (2000) Alteration of event related potentials in siblings discordant for schizophrenia. Schizophrenia Research, 41, 325-334.

Key, A.P.,Dove, G.O., Maguire, M.J. (2005) Linking brainwaves to the brain : an ERP primer.Developmental Neuropsychology, $27,183-215$.

Kim, M.S., Kang, S.S., Youn, T., et al (2003) Neuropsychological correlates of P300 abnormalities in patients with schizophrenia and obsessive-compulsivedisorder Psychiatry Research : Neuroimaging, 123, 109-123.

Kivircik, B.B., Yener, G.G., Alptekina, K., et al (2003) Eventrelated potentials and neuropsychological tests in obsessive-compulsive disorder. Progress in NeuroPsychopharmacology \& Biological Psychiatry, 27, 601-606.

Kornhuber, H.H. \& Deecke, L. (1978) An electrical sign of participation of the mesial "supplementary "motor cortex in human voluntary finger movements. Brain Research, 159, $473-476$.
Kutas, M., Lindamood, T., Hillyard, S. (1984) Word probability and event related potentials during sentence processing. In : Kornblum, S. \& Requin, J., (Eds.), Preparatory states and processes, Hillsdale, Erlbaum, pp. 217-238.

Leboyer,M.,Bellivier,F.,Nosten-Bertrand,M.,etal(1998)Psychiatric genetics:Search for phenotypes. Trends in Neurosciences, 21 , 102-105.

Light, G.A. \&Braff, D.L. (2003) Sensory gating deficits in schizophrenia, Can we parse the effects of medication, nicotine use, and changes in clinical status? Clinical Neuroscience Research, 3, 47-54.

Metzger, L.J., Carson, M.A., Paulus, L.A., et al (2002) Event-related potentials to auditory stimuli in female Vietnamnurse veterans with posttraumatic stress disorder. Psychophysiology, 39, 49-63.

Michie, P.T., Innes-Brown, H., Todd, J., et al (2002) Duration mismatch negativity in biological relatives of patients with schizophrenia spectrum disorders. Biological Psychiatry, 52, 749-758.

Myles-Worsley, M., Ord, L.M., Ngiralmau, H., et al (2007) The Palau early psychosis study: Neurocognitive functioning in high-risk adolescents. Schizophrenia Research, 89, 299-307.

Näätänen, R., Paavilainen, P., Reinikainen, K. (1998) Do eventrelated potentials to infrequent decrements in duration of auditory stimuli demonstrate a memory trace in man? Neuroscience letters, 107, 347-352.

Nanbu, M., Kurayama, T., Nakazawa, K., et al (2010) Impaired P50 suppression in fear extinction in obsessive-compulsive disorder. Progress in Neuro-Psychopharmacology \& Biological Psychiatry, 34, 317-322.

Osterhout, L. \& Holcomb, P.J. (1992) Event related brain potentials elicited by syntactic anomaly. Journal of Memory and Language, 31, 785-786.

Patrick, C.J., Bernat, E.M., Malone, S.M., et al (2006) P300 amplitude as an indicator of externalizing in adolescent males. Psychophysiology, 43, 84-92.

Pedersen, N.L., McClearn, G.E., Plomin, R., et al (1991) The Swedish Adoption/Twin Study of Aging : An update. Archives of General Medicine, 40, 7-20.

Peterson, N.N., Schroeder, C.E., Arezzo, J.C., et al (1995) Neural generators of early cortical somatosensory evoked potentials in the awake monkey. Electroencephalogrphy and Clinical Neurophysiology, 96, 248-260.

Pierson, A., Jouvent, R., Quintin, P., et al (2000) Information processing deficits in relatives of manic depressive patients. Psychological Medicine, 30, 545-555.

Polich, J. \& Hoffman, L.D. (1999) P300 handedness and corpus callosal size, gender, modality and task. International Journal of Psychophysiology, 33, 163-174

Polich, J. (1991) P300 in the evaluation in aging and dementia. In : Brunia, M., Mulder, G., Verbaten, M.N., (Eds.), Event Related Brain Potential Research (EEG Supplement 42), Amsterdam, Elsevier.

Price, G.W., Michie, P.T., Johnston, J., et al (2006) A multivariate Electrophysiological Endophenotype, from a Unitary Cohort, Shows Greater Research Utility than Any Single Feature in the Western Australian Family Study of Schizophrenia, Biological Psychiatry, 60, 1-10. 
Pritchard, W.S. (1986) Cognitive event related potentials correlates. Psychological Bulletin, 100, 43-66.

Rajender, G., Bhatia, M.S., Kanwal, K., et al (2011) Study of neurocognitive endophenotypes in drug naive obsessivecompulsive disorder patients, their first degree relatives and healthy controls. Acta Psychiatrica Scandinavica, 124, 152-161.

Rao, S. L. (2004) Cognitive Dysfunction and Cognitive Remediation in Schizophrenia, In : Mandal, M.K., Nizamie, S.H., (Eds.), Current Developments in Schizophrenia, Allied Publications PVT. Limited, New Delhi, pp. 304-340.

Roth, W.T. \& Cannon, E.H. (1972) Some features of the auditory evoked response in schizophrenics. Archives of General Psychiatry, 27, 466-471.

Roopesh, B. N., Rangaswamy, M., Kamarajan, C., et al (2010) Reduced Resource Optimization in Male Alcoholics : N400 in a Lexical Decision Paradigm. Alcoholism, Clinical and experimental Research, 34, 1905-1914.

Saitoh, O., Niwa, S., Hiramatsu, K., et al (1984) Abnormalities in late positive components of event-related potentials may reflect a genetic predisposition to schizophrenia. Biological Psychiatry, 19, 293-303.

Sanz, M., Molina, V., Martin-Loeches, M., et al (2001) Auditory event related potential and serotonin reuptake inhibitor treatment in obsessive-compulsive disorder patients. Psychiatry Research, 101, 75-81.

Sartory, G. \& Master, D. (1984) Contingent negative variation in obsessional-compulsive patients. Biological Psychiatry, 18, 253-267.

Schreiber, H., Stolz-Born, G., Kornhuber, H.H., et al (1992) Eventrelated potential correlates of impaired selective attention in children at high risk for schizophrenia. Biological Psychiatry, 32, 634-651.

Schulze, K., Hall, M.H., McDonald, C., et al (2005) Auditory P300 and duration mismatch negativity (MMN) as endophenotypes in psychotic bipolar disorder (BP). American Journal of Medical Genetics, 138B, 80.

Schulze, K., Hall, M.H., McDonald, C., et al (2007) P50 auditory evoked potential suppression in bipolar disorder patients with psychotic features and their unalected relatives. Biological Psychiatry, 62, 121-128.

Shelley, A.M., Ward, P.B., Catts, S.V., et al (1991) Mismatch negativity: An index of a preattentive processing deficit in schizophrenia. Biological Psychiatry, 30, 1059-1062.
Siddiqui, M.A.,Paul,I., Das, B., et al (2011) Role of Endophenotypes in Psychiatry. Rinpas Journal, 3, 245-264.

Siever, L.J. (2005) Endophenotypes in the personality disorders. Dialogues in Clinical Neuroscience, 7, 2.

Singh, R., Shukla, R., Dalal, P. K., et al (2000) P 300 Event-related potentials in depression. Indian Journal of Psychiatry, 42, 402-409.

Sur, S. \& Sinha, V.K. (2009) Event-related potential: An overview. Industrial Psychiatry Journal, 1, 70-73.

Tellegen, A., Lykken, T.D., Bouchard, T.J., et al (1988) Personality similarity in twins reared apart and together. Journal of Personality and Social Psychology, 54, 1031-1039.

Turetsky, B.I., Cannon, T.D., Gur, R.E. (2000) P300 subcomponent abnormalities in schizophrenia : III. Deficits In unaffected siblings of schizophrenic probands. Biological Psychiatry, $47,380-390$.

Waldo, M.C., Adler, L.E., Leonard, S., et al (2000) Familial transmission of risk factors in the first-degree relatives of schizophrenic people. Biological Psychiatry, 47, 231-239.

Waldo, M.C., Carey, G., Myles-Worsley, M., et al (1991) Codistribution of a sensory gating deficit and schizophrenia in multi-affected families. Psychiatry Research, 39, 257-268.

Walter, W.G. (1964) Slow potential waves in the human brain associated with expectancy, attention and decision. Archives of General Psychiatry, 206, 309-322.

Wang, Y., Adamczyk, A., Shugart, Y.Y., et al (2010) A screen of SLC1A1 for OCD-related alleles. American Journal of Medical Genetics Part B : Neuropsychiatric Genetics, 153, 675-679.

Wang, Y., Fang, Y., Chen, X., et al (2009) A follow-up study on features of sensory gating P50 in treatment-resistant depression. Chinese Medical Journal, 122, 2956-2960.

Winkler, I., Karmos, G., Näätänen, R. (1996) Adaptive modeling of the unattended acoustic environment reflected in the mismatch negativity event-related potential. Brain Research, $742,239-252$.

Winstanley, C.A., Dalley, J.W., Theobald, D.E., et al (2004) Fractionating impulsivity : Contrasting effects of central 5-HT depletion on different measures of impulsive behavior. Neuropsychopharmacology, 29, 1331-1343. 